\title{
A Modification to the WPC Model
}

Hossein Jahanshahloo*, Laima Spokeviciute

Leeds University Business School, Leeds, LS2 9JT, UK

\begin{abstract}
:
We modify the Barclay and Warner (1993) Weighted Price Contribution model - which measures market participants' contribution to price discovery process - to incorporate price movements that extend beyond the final price. We validate our model with an empirical illustration.
\end{abstract}

Keywords: Weighted Price Contribution, Price Discovery, High Frequency Trading. JEL classification: G1

${ }^{*}$ Corresponding author. Hossein Jahanshahloo, Tel:+441133439779

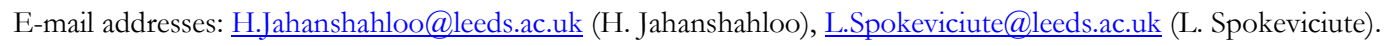




\section{Introduction}

One of the core functions of financial markets is the facilitation of price discovery - a process through which market players determine asset prices. A key issue concerning this process is how to empirically measure the impact that market players have on price discovery. One of the most commonly used methods is the application of the Weighted Price Contribution model (WPC), originally designed by Barclay and Warner (1993) to examine which trade moves prices first ${ }^{1}$ and adapted by Huang (2002) to measure individual market players' contribution to price discovery. The advantage of the WPC over other commonly used price discovery models is that it provides "a single measure that can be used in cross-sectional analyses" (Huang, 2002). However, one drawback of the WPC - especially given the increasing importance of high frequency trading - is that it may over- and/or underestimate the contribution of certain trades to price discovery. Specifically, overestimation occurs when prices are overshot in the direction, yet, outside the range of the total price movement; whereas the contributing factor of the trades that correct this overshoot is underestimated.

In this paper we present a Modified Weighted Price Contribution model (MWPC), which corrects for the above misestimation by incorporating the location of price movement relative to the final price. We further offer some empirical evidence on the validity of the MWPC.

\section{Original WPC, its Limitation and Empirical Validation}

\subsection{Original WPC}

Huang (2002) defines the WPC for dealer $j$ in stock $i$ as follows:

$$
W P C_{i, j}=\sum_{t=1}^{T}\left(\frac{\left|\Delta p_{t}^{i}\right|}{\sum_{t=1}^{T}\left|\Delta p_{t}^{i}\right|}\right)\left(\frac{\Delta p_{t}^{i, j}}{\Delta p_{t}^{i}}\right)
$$

where $\mathrm{T}$ is total number of periods in a day, $\Delta p_{t}^{i}$ is the price change of an asset $i$ in period $t$ and $\Delta p_{t}^{i, j}$ is the sum of all price changes that dealer $j$ is responsible for, which can be expressed as follows:

$$
\Delta p_{t}^{i, j}=\sum_{n=1}^{N_{t}}\left(p_{n}^{j}-p_{n-1}\right)
$$

where $N_{t}$ is the number of trades or quotes in period $t, p_{n}^{j}$ is the current price disseminated by dealer $j$ and $p_{n-1}$ is the previous price (by any dealer).

Consider a scenario of price formation, where, in a period $t$, the final price $\left(p_{f}^{j_{4}}\right)$ is higher than the start price $\left(p_{s}\right)$, as shown in Figure 1. Based on the WPC definition, any dealer that has disseminated a price

${ }^{1}$ For examples of the application of WPC model, see, Barclay and Hendershott, 2003; Huang, 2002; Madureira and Underwood, 2008; O’Hara et al., 2014. 
- relatively to the previous price - in the same (opposite) direction of the price movement from $p_{s}$ to $p_{f}^{j_{4}}$, has positively (negatively) contributed to the price discovery process. In this scenario the positive contributors are agents that move the prices from $p_{s}$ to $p^{j_{1}}, p^{j_{1}}$ to $p^{j_{2}}$ and $p^{j_{3}}$ to $p_{f}^{j_{4}}$, whereas the negative contribution is the price movement from $p^{j_{2}}$ to $p^{j_{3}}$.

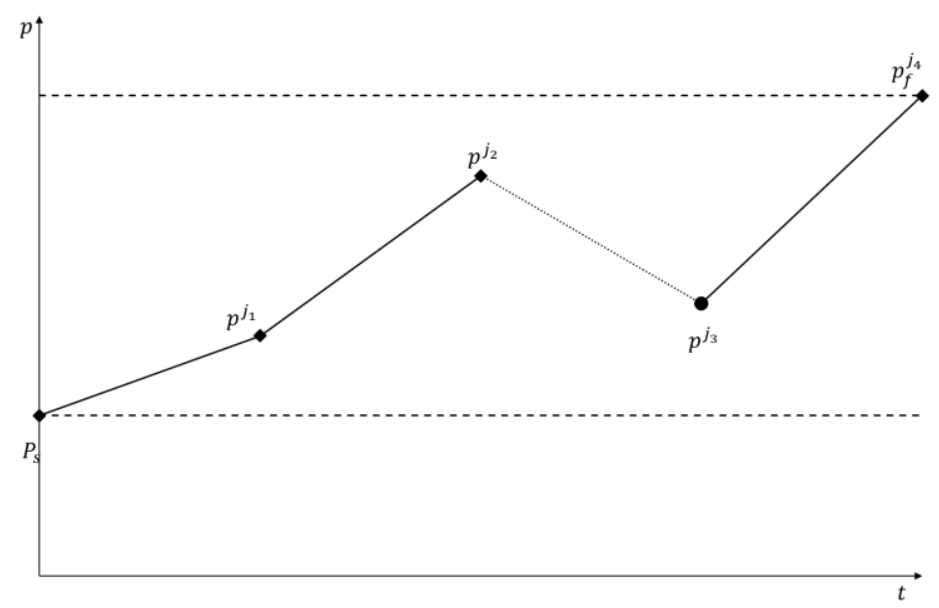

Figure 1. An illustration of a price formation process where the start price $\left(\mathrm{p}_{\mathrm{s}}\right)$ is lower than the final price $\left(p_{f}^{j_{4}}\right)$. The horizontal dash lines present the range of the start and final prices.

\subsection{WPC Limitation}

The scenario described above is ideal, as all the prices are within the range of the start and final prices. In financial markets, however, agents are heterogeneous, and an overshoot (undershoot) in prices may occur. In essence, the over and/or underestimation of the contribution to price discovery occurs if at least one of the prices - $p_{n-1}$ and/or $p_{n}^{j}$-are higher (lower) than the final price, where $p_{f}>p_{s}\left(p_{f}<p_{s}\right)$. We formalise the latter expression as follows:

$$
\begin{aligned}
& \operatorname{Max}\left\{p_{n-1}, p_{n}^{j}\right\}>p_{f}, \text { where } p_{f}>p_{s} \\
& \operatorname{Min}\left\{p_{n-1}, p_{n}^{j}\right\}<p_{f}, \text { where } p_{f}<p_{s}
\end{aligned}
$$

To illustrate the limitation of the WPC, we present a scenario of a single period price discovery process in Figure 2. As before, the start price $\left(p_{s}\right)$ is lower than the final price $\left(p_{f}^{j_{7}}\right)$ and the prices $p^{j_{1}}$ to $p_{f}^{j_{7}}$ are disseminated by dealers $j_{1}$ to $j_{7}$, respectively.

While the price changes disseminated by dealers $j_{1}, j_{2}$ and $j_{7}$ fall outside the ranges expressed in Eq. (3) the WPC misestimates the contribution to price discovery for dealers $j_{3}$ to $j_{6}$. Dealer $j_{3}\left(j_{6}\right)$ has traded in the same (opposite) direction of the price movement $\left(p_{s}\right.$ to $\left.p_{f}^{j_{7}}\right)$, however, at a price higher (lower) than the final price $p_{f}^{j_{7}}$. According to the WPC, a positive (negative) contribution to price discovery of $\frac{\left(p^{j_{3}}-p^{j_{2}}\right)}{\left(p_{f}^{j_{7}}-p_{s}\right)}$ 
$\left(\frac{\left(p^{j_{6}}-p^{j_{5}}\right)}{\left(p_{f}^{j_{7}}-p_{s}\right)}\right)$ is produced by $j_{3}\left(j_{6}\right)$. Consequently, the contribution of dealer $j_{3}\left(j_{6}\right)$ is overestimated (underestimated) due to the overshoot (partial price correction) of $\left|p^{j_{3}-} p_{f}^{j_{7}}\right|\left(\left|p^{j_{5}}-p_{f}^{j_{7}}\right|\right)$.

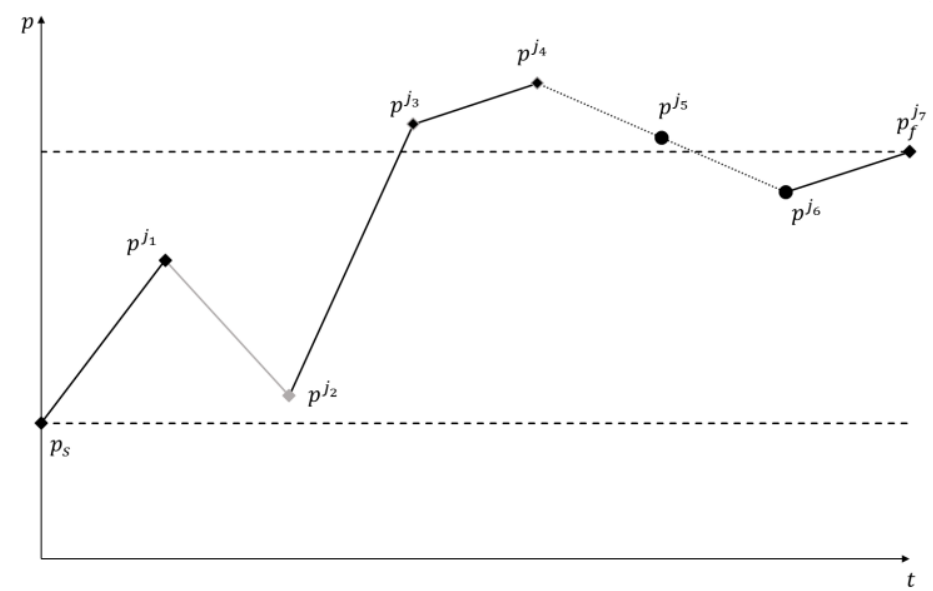

Figure 2. An illustration of a price formation process where the start price $\left(\mathrm{p}_{\mathrm{s}}\right)$ is lower than the final price $\left(\mathrm{p}_{\mathrm{f}}^{\mathrm{j}_{7}}\right)$. The horizontal dash lines present the range of the start and final prices.

Even more striking are the premises about dealers $j_{4}$ and $j_{5}$. Based on the WPC, a positive contributor is dealer $j_{4}$, who raised the price from $p^{j_{3}}$ to $p^{j_{4}}$, furthering the price from the final price by $\left|p^{j_{4}}-p^{j_{3}}\right|$, while dealer $j_{5}$, who has partially corrected the overreaction of dealer $j_{4}$ and has brought the price closer to its final price by $\left|p^{j_{5}}-p^{j_{4}}\right|$, obtains a negative WPC.

\subsection{WPC Limitation Empirical Validation}

Our premise for the requirement to modify the WPC is the notion that a considerable amount of price movements falls under the 'troublesome' price movements, as expressed in Eq. (3). We, therefore, present in Table 1 the summary statistics of these 'troublesome' price movements, based on high frequency one day $^{2}$ trade data for four stocks (Apple, Bank of America, J.P. Morgan, Microsoft) and the EUR:USD ${ }^{3}$ exchange rate. The summary statistics include the number of total ('troublesome') 1-minute intervals for the given asset on the day, shown in Column (I) ((II)). The results show that from the 390 (480) minute intervals observed for the stock (currency) data, a greater proportion incurred at least one 'troublesome' price movement.

We further report the mean, median and the maximum number of the 'troublesome' price movements within the samples of 'troublesome' minutes in Columns (III) to (V), respectively. The results show the mean (median) number of 'troublesome' price movements ranges from $23.09 \%$ to $31.50 \%$

\footnotetext{
${ }^{2}$ We obtain the trade data on equity and quote data on the currency pair for March 12 $2^{\text {th }}, 2018$ from Bloomberg and Thomson Reuters Eikon, respectively.

${ }^{3}$ Following Chaboud et al., (2014) we focus on the most active time of the market that is 8 to 16 GMT.
} 
(17.67\% to $25.76 \%)$ across different assets. Finally, in at least one of the 'troublesome' minutes, the ratio of 'troublesome' price movements exceeds $90 \%$ (80\%) for stock (currency) data.

Table 1.

Summary statistics of the 'troublesome' minutes and 'troublesome' price movements for a sample of high-frequency data on March $12^{\text {th }}, 2018$ at 1 -minute intervals.

\begin{tabular}{|c|c|c|c|c|c|}
\hline & (I) & (II) & (III) & (IV) & (V) \\
\hline & Total & Troublesome & Mean & Median & Max \\
\hline & \multicolumn{2}{|c|}{ Minutes } & \multicolumn{3}{|c|}{ Troublesome Price Movements } \\
\hline Apple & \multirow{4}{*}{390} & 330 & $26.94 \%$ & $19.03 \%$ & $96.02 \%$ \\
\hline Bank of America & & 258 & $25.42 \%$ & $17.67 \%$ & $93.98 \%$ \\
\hline J.P. Morgan & & 271 & $31.50 \%$ & $25.71 \%$ & $95.00 \%$ \\
\hline Microsoft & & 301 & $22.88 \%$ & $13.75 \%$ & $95.12 \%$ \\
\hline EUR:USD (Ask) & \multirow{2}{*}{480} & 262 & $24.43 \%$ & $18.83 \%$ & $83.00 \%$ \\
\hline EUR:USD (Bid) & & 255 & $23.09 \%$ & $18.24 \%$ & $83.06 \%$ \\
\hline
\end{tabular}

Overall, we show that the problem of over- and/or underestimation by WPC can materialize at different studied frequencies ${ }^{4}$, specifically when high volatility or price reversal exist.

\section{The Modified WPC}

\subsection{The Model}

In our Modified WPC (MWPC), rather than comparing the current price to the previous price, we measure the change in the distance to the final price. To do so, we first adjust the numerator of the right hand side of Eq. (1). More formally, we express the modified calculation of $\Delta p_{t}^{i, j}$ as follows:

$$
\nabla p_{t}^{i, j}=\sum_{n=1}^{N_{t}}(\underbrace{\left|p_{n-1}^{i}-p_{f}^{i}\right|}_{\text {distance I }}-|\underbrace{p_{n}^{i, j}-p_{f}^{i}}_{\text {distance II }}|),
$$

where $\nabla$ is used instead of $\Delta$ to highlight that convergence to the final price rather than the total price movement should be attributed to agent $j$, and Distance $I$ (II) shows the distance of $p_{n-1}$ to $p_{f}\left(p_{n}^{j}\right.$ to $p_{f}$ ). Consequently, the MWPC yields a negative value (contribution) to agent $j$ for price movements where $p_{n}^{j}$ is further from $p_{f}$ than the distance of $p_{f}$ to $p_{n-1}$. Whereas, whenever agent $j$ reduces the distance to $p_{f}$ a positive contribution to price discovery will be obtained.

Note here, that with our modification, we can now ignore the direction of the start to final price movement. This is because we propose to focus on price convergence to the final price, rather than the actual price change. In addition MWPC does not violate the prerequisite of the WPC that all agents' contribution to price discovery sums up to $100 \%$. In essence, we change the final and start price difference $\left(\Delta p_{t}^{i}\right)$ to its absolute value $\left(\left|\Delta p_{t}^{i}\right|\right)$. The formal expression of the MWPC is as follows:

\footnotetext{
${ }^{4}$ We extend our analysis using alternative to 1 -minute time intervals and find similar results, however, due to brevity, do not report these results.
} 


$$
M W P C_{i, j}=\sum_{t=1}^{T}\left(\frac{\left|\Delta p_{t}^{i}\right|}{\sum_{t=1}^{T}\left|\Delta p_{t}^{i}\right|}\right)\left(\frac{\nabla p_{t}^{i, j}}{\left|\Delta p_{t}^{i}\right|}\right)=\sum_{t=1}^{T}\left(\frac{\nabla p_{t}^{i, j}}{\sum_{t=1}^{T}\left|\Delta p_{t}^{i}\right|}\right)
$$

\subsection{Empirical MWPC Illustration}

To illustrate the economic significance of the modification to the WPC, we compare the price discovery estimates derived by the two models for ten most active market dealers in the EUR:USD currency pair 5 . We report in Table 2 the mean (median) absolute differences between the estimates for ask and bid price discovery separately, with t-values (z-values) of the mean (median) equality tests provided in the parentheses below.

Table 2.

Summary of the absolute differences between WPC and MWPC estimates for the top 10 most active dealers in EUR:USD, on March $12^{\text {th }}, 2018$ at 1-minute frequency.

\begin{tabular}{lccc}
\hline & & Mean $(\%)$ & Median $(\%)$ \\
\hline \multirow{3}{*}{ EUR:USD } & Ask & $6.53^{* * *}$ & $5.61^{* * *}$ \\
& & $(4.22)$ & $(2.81)$ \\
& \multirow{2}{*}{ Bid } & $5.24^{* *}$ & $2.44^{* * *}$ \\
& & $(2.73)$ & $(2.80)$ \\
\hline
\end{tabular}

The results provide evidence that the two models yield significantly different results, as indicated by statistically significant mean and median differences for both, ask and bid prices. In essence, we show that the MWPC corrects the over-/under-estimation of WPC. Unreported tests further show that the results are robust to the use of various settings (i.e. using different intervals, including all dealers in the sample, etc.).

\section{Conclusions}

The emergence of high frequency trading has changed the nature of the price discovery process. How this phenomena affected the application of the Weighted Price Contribution model, however, has been unexplored. In this paper we re-examine the WPC and theoretically and empirically show that when a dealer submits a price beyond the final price the WPC over- and/or underestimates a market participant's contribution to price discovery. This is especially more likely to occur when the number of trades, coupled with higher price volatility and growth in price reversal trends, increases. We address this issue with our Modified WPC model by estimating the contribution to price discovery based on convergence of prices towards the final price. Using high frequency data, we further our argument with some empirical evidence on the different estimates the two models yield for the top 10 most active dealers in the EUR:USD currency pair.

${ }^{5}$ We chose the currency pair data for the illustration because it contains dealers' identity. This allows us to compare WPC and MWPC for individual dealers. 


\section{References}

Barclay, M. J. and Hendershott, T., 2003. "Price Discovery and Trading After Hours", The Review of Financial Studies, 16(4), pp. 1041-1073.

Barclay, M. J. and Warner, J.B., 1993. "Stealth trading and volatility: Which trade moves prices?”, Journal of Financial Economics, 34(3), pp. 281-350.

Chaboud, A. P., Chiquoine, B., Hajlmarsson, E. and Vega, C., 2014. "Rise of The Machines: Algorithmic Trading in the Foreign Exchange Market", The Journal of Finance 69(5), pp. 2045-2084.

Huang, R. D., 2002. “The Quality of ECN and Nasdaq Market Maker Quotes”, The Journal of Finance, 57(3), pp. 12851319.

Madureira, L. and Underwood, S., 2008. "Information, sell-side research, and market making", Journal of Financial Economics, 90(2), pp. 105-126.

O’Hara, M., Yao, C. and Ye, M., 2014. "What's Not There: Odd Lots and Market Data”, The Journal of Finance, 69(5), pp. 2199-2236. 